\title{
Feasibility and Safety of Transcutaneous Vagus Nerve Stimulation Paired with Notched Music Therapy for the Treatment of Chronic Tinnitus
}

\author{
Hyun Joon Shim, Min Young Kwak, Yong-Hwi An, Dong Hyun Kim, Yun Jin Kim, and Hyo Jung Kim \\ Department of Otorhinolaryngology, Eulji Medical Center, Eulji University School of Medicine, Seoul, Korea
}

Received September 12, 2015

Revised November 17, 2015

Accepted November 30, 2015

\section{Address for correspondence Hyun Joon Shim, MD, PhD \\ Department of Otorhinolaryngology, Eulji Medical Center, \\ Eulji University School of Medicine, 68 Hangeulbiseok-ro, Nowon-gu, Seoul 01830, Korea \\ Tel $+82-2-970-8276$ \\ Fax +82-2-970-8275 \\ E-maileardoc11@naver.com}

\begin{abstract}
Background and Objectives: A recent study demonstrated that tinnitus could be eliminated by vagus nerve stimulation (VNS) paired with notched sounds in a rat tinnitus model. The aims of this clinical study were to investigate the effects and safety of transcutaneous VNS (tVNS) by patch-type electrode paired with notched music for treating chronic tinnitus. Subjects and Methods: Thirty patients with refractory chronic tinnitus for $>12$ months were included in this study. A patch-type electrode was attached to the auricular concha of the patient's left ear and tVNS was performed for 30 min (pulse rate $25 \mathrm{~Hz}$, pulse width $200 \mu \mathrm{s}$, and amplitude 1-10 $\mathrm{mA}$ ) using a transcutaneous electric nerve stimulation eco2. During tVNS, the patients listened to notched music cleared of the frequency spectrum corresponding to the tinnitus with a 0.5 octave notch width. Results: After 10 treatment sessions, $15 / 30$ patients $(50 \%)$ reported symptom relief in terms of a global improvement questionnaire. The mean tinnitus loudness (10-point scale) and the mean tinnitus awareness score (\%) improved significantly from $6.32 \pm 2.06$ to $5.16 \pm 1.52$ and from $82.40 \pm 24.37 \%$ to $65.60 \pm 28.15 \%$, respectively (both $p<0.05$ ). None of the patients had any specific side effects, such as changes in heart rate or blood pressure. Conclusions: This study has demonstrated the feasibility and safety of tVNS paired with notched music therapy in patients with chronic tinnitus, with the use of a pad-type electrode attached to the auricular concha.
\end{abstract}

J Audiol Otol 2015;19(3):159-167

KEY WORDS: Vagus nerve stimulation - Transcutaneous electrical nerve stimulation . Music therapy $\cdot$ Tinnitus.

\section{Introduction}

Neuromodulation by vagus nerve stimulation (VNS) stimulates the cholinergic nucleus basalis, generating powerful and prolonged changes in cortical organization [1]. Therefore, electrical stimulation of the vagus nerve could increase alertness [2,3], and enhance the recovery of motor and cognitive functions in animal models of traumatic brain injury [4]. VNS has been used to treat a variety of maladaptive neuronal plasticity disorders such as epilepsy, depression, and chronic

This is an Open Access article distributed under the terms of the Creative Commons Attribution Non-Commercial License (http://creativecommons. org/licenses/by-nc/3.0/) which permits unrestricted non-commercial use, distribution, and reproduction in any medium, provided the original work is properly cited. pain [2-4]. There is abundant data showing the safety of cervical vagal nerve stimulation using implanted electrodes, and the United States Food and Drug Administration has approved VNS for the treatment for drug-resistant epilepsy and depression.

Maladaptive neuronal plasticity of the central auditory system triggered by preceding cochlear damage is a general neurophysiologic model that can explain selective tinnitus following cochlear damage. Reduced signal transduction from damaged hair cells may cause a reduction in the lateral inhibition of the central auditory system, followed by increased synchronous firing or spontaneous activity in the auditory neurons at the edge of the characteristic frequency $[5,6]$. Therefore, the onset of tinnitus is dependent on the plastic changes in the central auditory system induced by deafferentation rather 
than the degree of end-organ damage. In this context, neuromodulation by VNS has emerged as a new treatment option for tinnitus. In fact, in recent years, various other neuromodulation strategies have been used to treat tinnitus, including transcranial magnetic stimulation of the auditory cortex or cingulate cortex, transcranial direct current stimulation, transcutaneous electric nerve stimulation (TENS), and implantation of electrodes into the auditory cortex [7-10]. However, these approaches only temporarily disrupted ongoing activity and did not provide long-term control of tinnitus.

A new therapeutic concept of neuromodulation in which VNS is paired with a specific tone was recently reported [11]. The authors demonstrated that pairing brief pulses of cervical VNS with a variety of tones that excluding the tinnitus frequency had the effect of increased frequency selectivity and decreased synchronization, and completely eliminated tinnitus in noise-exposed rats. However, surgical implantation of an electrode into the neck to treat tinnitus is a difficult procedure in human owing to its invasiveness, surgical risks, and high costs. Moreover, side effects such as disturbed cardiac rhythm or coughing may occur during stimulation. Therefore, transcutaneous VNS (tVNS) is emerging as an alternative treatment option. Transcutaneous electrical stimulation of the cutaneous branch of the vagus nerve, also called Arnold's nerve, in the external ear was proposed as an alternative, noninvasive modality for VNS [12]. A functional magnetic resonance imaging (fMRI) and electroencephalography (EEG) study revealed that the central vagal pathway could be stimulated by tVNS $[13,14]$. In the current study, we used tVNS instead of cervical VNS and aimed to investigate the effects and safety of tVNS by the patch-type electrode paired with notched music for treating refractory chronic tinnitus.

\section{Subjects and Methods}

\section{Patients}

Thirty patients ( 21 males, 9 females, mean age $58.47 \pm$ 15.85 years) with idiopathic subjective tinnitus were included in this study. Patients were eligible if they met the following criteria: 1) symptom duration of $>12$ months; 2) discomfort affecting their daily lives; 3 ) ongoing symptoms refractory to pharmacotherapy or tinnitus-retraining therapy; 4) consistent tinnitus frequency measured by pitch matching in three tests performed on different days; 5) no history of otologic disease; 6) no history of ototoxic drug use; 7) no accompanying retrocochlear tumor, otitis media, or Meniere's disease; and 8) no history of temporomandibular joint or cervical problems. All of the patients underwent audiologic tests including pure-tone audiometry, tinnitus pitch matching, loudness matching, imped- ance audiometry, auditory brainstem response tests, and otoacoustic emission tests. Tinnitus pitch matching was performed three times on different days, and we excluded patients whose pitch matching data were not consistent in all three tests. We excluded patients with subjective tinnitus accompanied by sudden sensorineural hearing loss of $\geq 30 \mathrm{~dB}$ on three contiguous audiometric frequencies in $<72 \mathrm{~h}$. We also excluded patients who were suspected of having objective tinnitus or somatic tinnitus based on physical examination of the head and neck. Electrocardiograms and blood pressure were measured to exclude patients with possible cardiac problems, and we excluded patients with cardiac arrhythmia or asthma because these may present safety issues for VNS.

Of the 30 patients, 22 had unilateral tinnitus and 8 had bilateral tinnitus. In patients with unilateral tinnitus, the right ear was affected in 13 patients and the left ear was affected in 9 patients. Before treatment, the mean pure-tone average was $29.50 \pm 18.22 \mathrm{~dB}$ HL for the right ear and $32.83 \pm 25.51$ $\mathrm{dB}$ HL for the left ear. This study was approved by the Institutional Review Board of the Eulji Medical Center, South Korea (MD_IRB_2015-12).

\section{Electrical and sound stimuli}

Each patient underwent a total of 10 sessions $(30 \mathrm{~min} / \mathrm{ses}-$ sion), and each session was performed at intervals of 1-4 days depending on the patient's condition. The patch-type electrode was attached to the auricular concha of the left ear and tVNS was performed for $30 \mathrm{~min}$ (pulse rate $25 \mathrm{~Hz}$, pulse width $200 \mu$ s, and intensity $1-10 \mathrm{~mA}$ ) using a TENS eco2 (Schwa-medico, Ehringshausen, Germany). We attached a silicon electrical pad ( $2 \mathrm{~cm}$ in diameter) as closely as possible to the concha and outer part of the external meatus. The intensities of stimuli used in each session were set at the highest levels the patient could endure. During tVNS, the patients listened to tailored notched music after removal of the frequency spectrum corresponding to the tinnitus at a $1 / 2$ octave notch width. The patients selected their favorite piece of music from four samples of classical music.

\section{Outcome measures}

The patients completed four different questionnaires before treatment and again immediately after the end of the 10th session. Subjective improvements in tinnitus were measured by determining the changes in the following four variables: 1) global improvement (GI) index in subjective tinnitus severity (Table 1) [15]; 2) Visual Analogue Scale (VAS) of tinnitus loudness (a 10-point scale where $0=$ no tinnitus and $10=$ the loudest tinnitus imaginable); 3) tinnitus awareness score (patients were asked to describe the percentage of time 
aware of tinnitus over 1 day at intervals of $10 \%$ ); and 4) Tinnitus Handicap Inventory (THI) [16]. Thirty patients completed the GI index after treatment, 25 patients completed the VAS of tinnitus loudness and tinnitus awareness score after treatment because 5 patients did not take the pre-treatment questionnaires, and 24 patients completed the THI after treatment because one more patient refused to reply to the pretreatment THI.

\section{Statistical analyses}

Data were analyzed using SPSS statistical software (SPSS, version 22.0 for Windows; SPSS Inc., Chicago, IL, USA). Wilcoxon's signed-ranks test were used to compare the pre- and post-treatment mean VAS of tinnitus loudness, mean tinnitus awareness score, and mean THI score. To determine which factors could influence symptom improvement, multiple linear regression analyses of the post-treatment GI index were performed with various factors: pure-tone average of the affected side, tinnitus duration, initial VAS of tinnitus loudness, initial tinnitus awareness score, and initial THI. Correlations between the time to symptom relief in terms of the number of treatment sessions and the variables of GI index, the improvement (post score-pre score) in VAS of the tinnitus loudness, tinnitus awareness score, and THI were analyzed using Pearson's correlation coefficient.

\section{Results}

\section{Characteristics of tinnitus}

The mean duration of tinnitus was $42.40 \pm 45.64$ months (range 12-240 months), and other characteristics of tinnitus

Table 1. Global improvement in tinnitus severity

\begin{tabular}{cl}
\hline Grade & Markedly worse \\
\hline 1 & Somewhat worse \\
3 & Marginally worse \\
4 & Same \\
5 & Marginally better \\
6 & Somewhat better \\
7 & Markedly better \\
8 & Completely disappeared \\
\hline
\end{tabular}

and patients are listed in Table 2. In cases of bilateral tinnitus, characteristics of dominant side were included.

\section{GI index}

When improvement was defined as GI $\geq$ grade 5 , of the 30 patients with refractory chronic tinnitus, 15 patients $(50 \%)$ reported symptom relief in terms of the GI index after 10 treatment sessions. In these 15 patients, the mean time to symptom relief was 4.60th \pm 3.20 sessions (range $1-10$ sessions) (Table 3 ). However, no one reported complete elimination of tinnitus. The mean GI index was $4.63 \pm 0.89$, and $6 / 30$ patients $(20.0 \%)$ reported "somewhat better" in symptoms (grade 6), $9 / 30$ patients $(30.0 \%)$ reported "marginally better" (grade 5 ), $13 / 30$ patients $(43.3 \%)$ reported "same" (grade 4), and 2/30 patients $(6.6 \%)$ reported "marginally worse" (grade 3 ) in symptoms, as shown in Fig. 1.

\section{Changes in subjective tinnitus loudness}

Twenty-five patients completed the follow-up questionnaire on subjective tinnitus loudness. The mean VAS of tinnitus loudness decreased significantly (i.e., improved) from $6.32 \pm 2.06$ at baseline to $5.16 \pm 1.52$ after tVNS paired with notched music therapy ( $p=0.005)$. In $16 / 25$ patients $(64.0 \%)$, the VAS of tinnitus loudness decreased from baseline to after tVNS, with a change from pre-treatment to post-treatment (post score-pre score) of -4 in 3 patients, -3 in 3 patients, -2 in 4 patients, and -1 in 6 patients. Five patients $(20.0 \%)$ reported no change in subjective loudness and 4 patients $(16.0 \%)$ reported an increase in loudness $(+1$ in 3 patient and +3 in 1 patients) (Fig. 1).

\section{Changes in subjective tinnitus awareness}

Twenty-five patients completed the follow-up questionnaire on subjective tinnitus awareness. The mean tinnitus awareness score (\%) decreased significantly from $82.40 \pm$ $24.37 \%$ at baseline to $65.60 \pm 28.15 \%$ after treatment $(p=$ $0.020)$. The tinnitus awareness score decreased (i.e., improved) in $13 / 25$ patients $(52.0 \%$ ), was unchanged in $8 / 25$ patients $(32.0 \%)$, and increased in $4 / 25$ patients (16.0\%) (Fig. 1). The mean change in the tinnitus awareness score in the 13 patients who reported a reduction in this variable was $41.54 \pm$ $21.15 \%$ (range $10-80 \%$ ).

Table 2. Characteristics of tinnitus in all patients

\begin{tabular}{ccccccc}
\hline Pitch $(\mathrm{kHz})$ & Loudness $(\mathrm{dB} \mathrm{SL})$ & Duration (month) & THI score & VAS of tinnitus loudness & TAS (\%)* & BDI \\
\hline $3.93 \pm 2.94$ & $4.33 \pm 2.20$ & $42.40 \pm 45.64$ & $41.50 \pm 29.64$ & $6.32 \pm 2.06$ & $82.40 \pm 24.37$ & $12.92 \pm 8.62$ \\
\hline
\end{tabular}

In cases of bilateral tinnitus, characteristics of dominant side were included. *tinnitus awareness score is defined as the percentage of the time the patient is aware of tinnitus for a day. THI: Tinnitus Handicap Inventory, VAS: Visual Analogue Scale, BDI: Beck Depression Inventory 
Table 3. Treatment results in individual questionnaires

\begin{tabular}{|c|c|c|c|c|c|c|c|c|c|c|c|c|}
\hline \multirow[t]{2}{*}{ Subject no. } & \multirow{2}{*}{$\begin{array}{l}\text { Sex/ } \\
\text { age }\end{array}$} & \multirow[t]{2}{*}{$\mathrm{Gl}$} & \multirow{2}{*}{$\begin{array}{l}\text { Time to } \\
\text { symptom relief* }\end{array}$} & \multicolumn{3}{|c|}{ VAS of tinnitus loudness } & \multicolumn{3}{|c|}{ Tinnitus awareness score (\%) } & \multicolumn{3}{|c|}{$\begin{array}{l}\text { Tinnitus Handicap } \\
\text { Inventory score }\end{array}$} \\
\hline & & & & Pre & Post & Post-pre & Pre & Post & Post-pre & Pre & Post & Post-pre \\
\hline 1 & $M / 57$ & 4 & & 4 & 4 & 0 & 80 & 50 & -30 & 28 & 12 & -16 \\
\hline 2 & $M / 80$ & 6 & $1^{\text {st }}$ & 5 & 4 & -1 & 100 & 20 & -80 & 30 & 10 & -20 \\
\hline 3 & $M / 53$ & 4 & & 5 & 5 & 0 & 70 & 30 & -40 & 6 & 10 & 4 \\
\hline 4 & $M / 64$ & 5 & $2^{\text {nd }}$ & 6 & 6 & 0 & 100 & 50 & -50 & 26 & 46 & 20 \\
\hline 5 & $M / 67$ & 4 & & 8 & 5 & -3 & 100 & 100 & 0 & 30 & 14 & -16 \\
\hline 6 & $\mathrm{~F} / 51$ & 4 & & ND & & & ND & & & ND & & \\
\hline 7 & $M / 62$ & 4 & & 5 & 4 & -1 & 80 & 80 & 0 & 18 & 18 & 0 \\
\hline 8 & $M / 23$ & 5 & $5^{\text {th }}$ & 7 & 5 & -2 & 100 & 100 & 0 & 62 & 44 & -18 \\
\hline 9 & $M / 41$ & 5 & $9^{\text {th }}$ & 9 & 6 & -3 & 70 & 70 & 0 & 72 & 60 & -12 \\
\hline 10 & $M / 58$ & 5 & $10^{\text {th }}$ & 5 & 4 & -1 & 80 & 60 & -20 & 22 & 22 & 0 \\
\hline 11 & $M / 81$ & 5 & $6^{\text {th }}$ & 4 & 3 & -1 & 40 & 100 & 60 & 14 & 14 & 0 \\
\hline 12 & $M / 73$ & 4 & & 4 & 5 & 1 & 100 & 100 & 0 & 44 & 56 & 12 \\
\hline 13 & $\mathrm{~F} / 65$ & 6 & $5^{\text {th }}$ & 7 & 5 & -2 & 100 & 50 & -50 & 70 & 18 & -52 \\
\hline 14 & $M / 64$ & 4 & & 9 & 9 & 0 & 100 & 100 & 0 & 78 & 70 & -8 \\
\hline 15 & $M / 56$ & 4 & & ND & & & ND & & & ND & & \\
\hline 16 & $F / 69$ & 4 & & 5 & 5 & 0 & 20 & 40 & 20 & 18 & 92 & 74 \\
\hline 17 & $F / 22$ & 4 & & ND & & & ND & 20 & & ND & & \\
\hline 18 & $M / 51$ & 3 & & 5 & 8 & 3 & 100 & 100 & 0 & 16 & 54 & 38 \\
\hline 19 & $\mathrm{~F} / 76$ & 5 & $7^{\text {th }}$ & 6 & 5 & -1 & 100 & 100 & 0 & 100 & 32 & -68 \\
\hline 20 & $M / 40$ & 5 & $1^{\text {st }}$ & 10 & 6 & -4 & 100 & 90 & -10 & 98 & 46 & -52 \\
\hline 21 & $M / 57$ & 4 & & $N D$ & & & ND & & & ND & & \\
\hline 22 & $\mathrm{~F} / 60$ & 6 & $10^{\text {th }}$ & 9 & 5 & -4 & 70 & 80 & 10 & 14 & 18 & 4 \\
\hline 23 & $M / 70$ & 4 & & 3 & 4 & 1 & 70 & 30 & -40 & 44 & 47 & 3 \\
\hline 24 & $F / 37$ & 3 & & 5 & 6 & 1 & 80 & 40 & -40 & 38 & 70 & 32 \\
\hline 25 & $M / 64$ & 6 & $3^{\text {rd }}$ & 10 & 6 & -4 & 100 & 20 & -80 & 98 & 44 & -54 \\
\hline 26 & $M / 72$ & 6 & $2^{\text {nd }}$ & 5 & 3 & -2 & 80 & 50 & -30 & 10 & 20 & 10 \\
\hline 27 & $F / 61$ & 5 & $4^{\text {th }}$ & 9 & 8 & -1 & 100 & 80 & -20 & 26 & 28 & 2 \\
\hline 28 & M/82 & 6 & $3^{\text {rd }}$ & 7 & 5 & -2 & 20 & 50 & 30 & 34 & 6 & -28 \\
\hline 29 & $M / 67$ & 4 & & ND & & & ND & & & ND & & \\
\hline 30 & M/35 & 5 & $1^{\text {st }}$ & 6 & 3 & -3 & 100 & 50 & -50 & ND & & \\
\hline Average & & 4.63 & $4.60^{\text {th }}$ & 6.32 & 5.16 & -1.16 & 82.40 & 65.60 & -16.80 & 41.50 & 35.46 & -6.04 \\
\hline Standard de & viation & 0.89 & 3.20 & 2.06 & 1.52 & 1.77 & 24.37 & 28.15 & 32.75 & 29.64 & 23.30 & 31.58 \\
\hline
\end{tabular}

*time to symptom relief in terms of the number of treatment sessions in 15 subjects who reported symptom relief in the Gl index. VAS: Visual Analogue Scale, Gl: global improvement, ND: not determined

\section{Changes in THI score}

Twenty-four patients completed the THI after treatment. Although the mean THI score decreased from $41.50 \pm 29.64$ at baseline to $35.46 \pm 23.30$ after treatment, this change was not statistically significant $(p=0.339)$. The THI score decreased from baseline to after treatment in $11 / 24$ patients $(45.8 \%)$, was unchanged in 3 patients $(12.5 \%)$, and worsened in 10 patients (41.7\%) (Fig. 1).

\section{Multiple linear regression analyses}

About $45.7 \%$ of $\mathrm{R}^{2}$ for the model was explained by clinically relevant factors including pure-tone average of the af- fected side, duration, initial VAS of tinnitus loudness, initial tinnitus awareness score, and initial THI. The GI index after treatment, as the outcome variables for symptom improvement, was significantly associated with only tinnitus $(p=0.007)$ (Table 4).

\section{Correlation analyses}

Fig. 2 shows that the time to symptom relief was not significantly correlated with the improvement (post score-pre score) in initial VAS of the tinnitus loudness, tinnitus awareness score, and THI, or with the GI index in the 15 patients who reported improvements in their symptoms (all $p>0.05$ ). 


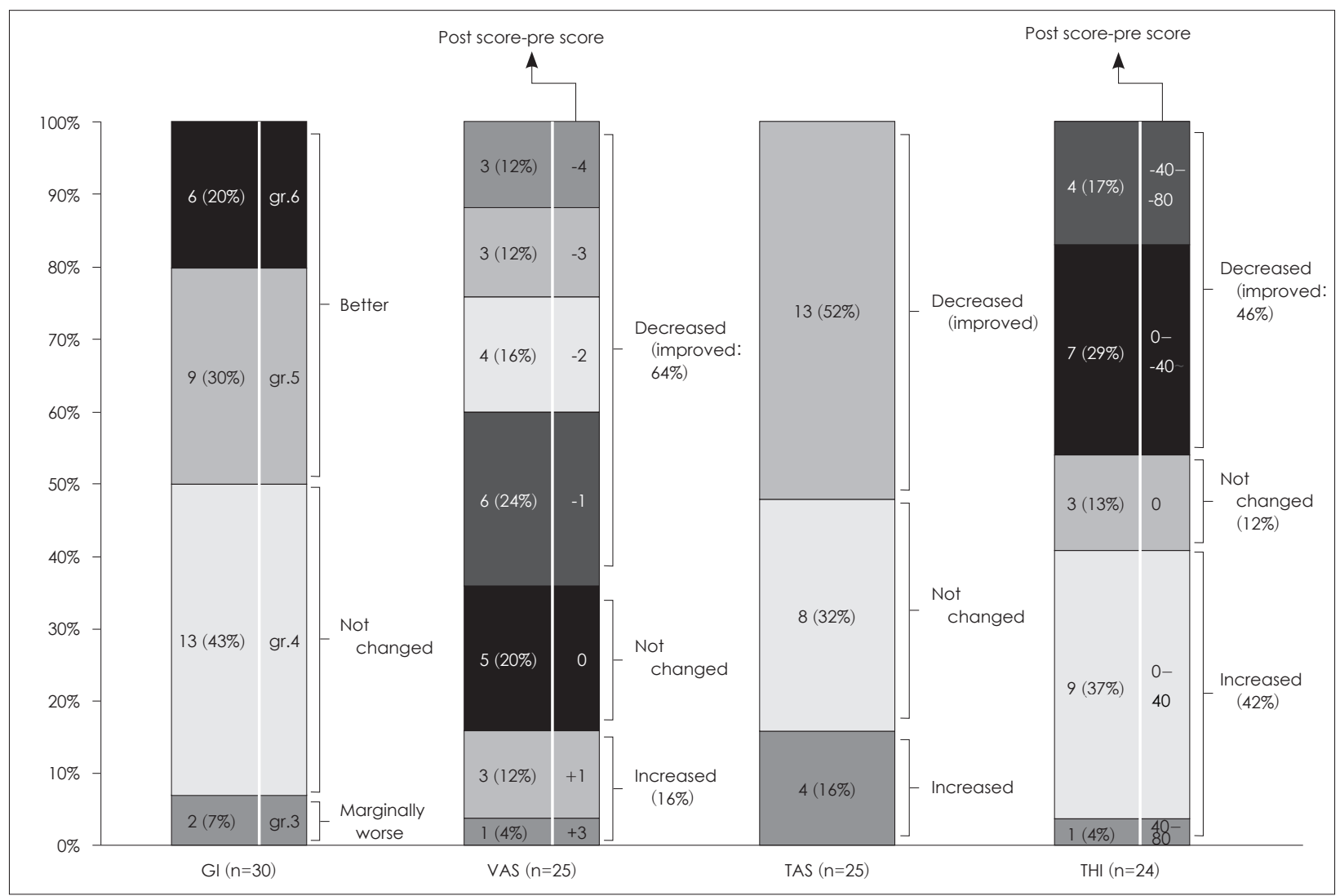

Fig. 1. Global improvement (GI) index and change in Visual Analogue Scale (VAS) of tinnitus loudness (10-point), tinnitus awareness score (TAS, \%), and Tinnitus Handicap Inventory (THI) score immediately after the end of the 10th session. Of the 30 patients, 15 patients reported an improvement in symptom (grade 5 and 6 ), 13 patients reported no change (grade 4), and 2 patients reported mild worsening of symptoms (grade 3 ). In 16/25 patients (64.0\%), the VAS of tinnitus loudness decreased (i.e., improved), five patients (20.0\%) reported no change in subjective loudness and 4 patients $(16.0 \%)$ reported an increase in loudness. The tinnitus awareness score decreased (i.e., improved) in $13 / 25$ patients $(52.0 \%)$, was unchanged in $8 / 25$ patients $(32.0 \%)$, and increased in $4 / 25$ patients $(16.0 \%)$. The THI score decreased from baseline to after treatment in $11 / 24$ patients $(45.8 \%)$, was unchanged in 3 patients (12.5\%), and worsened in 10 patients $(41.7 \%)$.

Table 4. Multiple linear regression analyses of various factors and global improvement after tinnitus treatment

\begin{tabular}{|c|c|c|c|c|}
\hline & Factor & Standardized $\beta$ coefficient & $\mathrm{R}^{2}$ & $p$ value \\
\hline \multirow[t]{5}{*}{ Global improvement } & PTA & -0.065 & 0.457 & 0.717 \\
\hline & Duration & 0.539 & & $0.007^{*}$ \\
\hline & Initial VAS of tinnitus loudness & 0.370 & & 0.093 \\
\hline & Initial tinnitus awareness & -0.232 & & 0.242 \\
\hline & Initial THI & 0.203 & & 0.384 \\
\hline
\end{tabular}

*p<0.05. PTA: pure-tone average, VAS: Visual Analogue Scale, THI: Tinnitus Handicap Inventory

\section{Safety}

None of the patients experienced specific side effects, such as changes in their heart rate or blood pressure.

\section{Discussion}

In this study, we enrolled patients with idiopathic subjective tinnitus lasting for $>12$ months (mean $42.40 \pm 45.64$ months) despite conventional therapies, including pharmaco- therapy or tinnitus retraining therapy. After 10 sessions of tVNS paired with notched music, the GI index, which consists of eight grades reflecting the overall change in subjective symptom severity, improved in $50 \%$ of the patients. The mean tinnitus loudness and awareness scores also improved significantly after treatment.

Many previous studies reported that maximal improvement rates from the placebo effect were $30 \%$ to $40 \%$ when using various questionnaires [17-19]. In the current study, the im- 


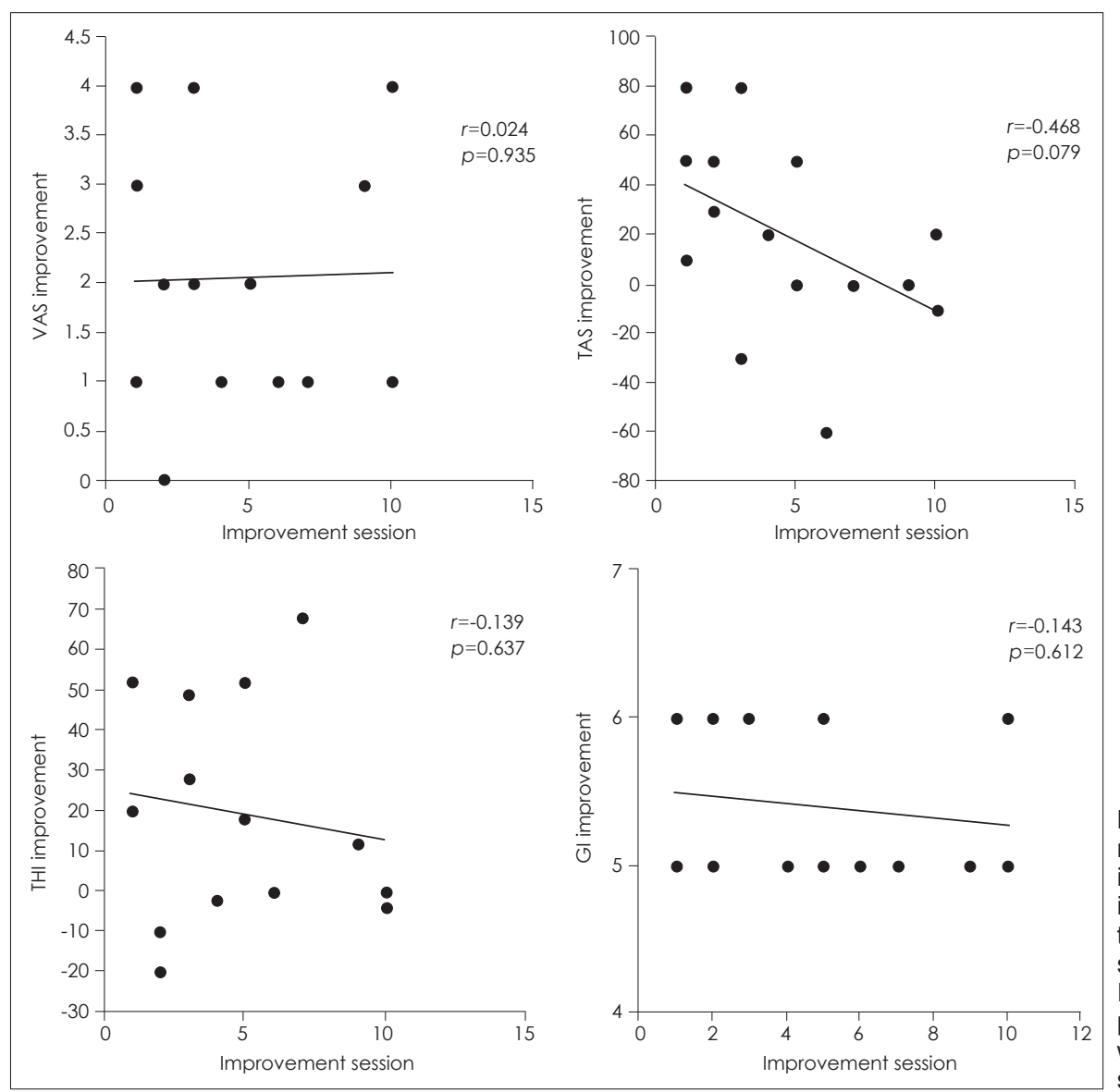

Fig. 2. Time to symptom relief was not significantly correlated with the improvement (post score-pre score) in Visual Analogue Scale (VAS) of the tinnitus loudness, tinnitus awareness score (TAS), and Tinnitus Handicap Inventory (THI), or with the global improvement $(\mathrm{Gl})$ index in the 15 patients who reported improvements in their symptoms (all $p>0.05$ ). provement rates were $50 \%, 64 \%$, and $52 \%$ in GI index, subjective tinnitus loudness and tinnitus awareness respectively, and all of them far exceeded $40 \%$ of the maximal placebo effect. On the basis of this fact, we believe that tVNS paired with notched music has a high possibility of a therapeutic effect exceeding the placebo effect in patients with refractory chronic tinnitus, although we did not compare to controls. The only variable that was not significantly improved was the THI score, which may be due to the relatively short study duration. Because we enrolled patients with refractory chronic tinnitus, it may take longer to observe a significant reduction in the THI score. Additionally, the THI comprises several questions covering functional, emotional, and catastrophic subscales, which are more complex than the questions in the other questionnaires.

A recent animal study laid the foundation for using VNS with tone to treat tinnitus [11]. The authors reported that pairing a brief burst of VNS with a $9 \mathrm{kHz}$ or $19 \mathrm{kHz}$ tone in normal rats significantly increased the fraction of the primary auditory cortex site responding to these frequencies. This implies that the combined tones could lead the VNS-induced neural responses to the exact tonotopic maps in the auditory cortex, and concentrate the effects of VNS to the target region. They demonstrated restoration of the distorted tonotopic maps in the auditory cortex and elimination of tinnitus under behavioral observation in all cases. Unlike the animal study [11], all patients did not get the benefit in the current study. We could expect several reasons for the difference. One of the reasons could be the difference of onset time of treatment (over 12 months after tinnitus in our subjects vs. 4 weeks after noise exposure to make tinnitus in the animal study). Indeed, several studies demonstrated that early onset of neuromodulation made better results [20,21]. Another possible reason is the difference of device for VNS (tVNS in our study vs. direct cervical stimulation via surgically implanted electrode in the animal study). Moreover, the rat received more intensive treatment, 2.5 hours per day for 20 day than our treatment. The inter-species characteristic could be another possible reason. De Ridder, et al. [22] have tried cervical VNS with an implanted electrode to treat tinnitus in humans, and they also demonstrated only 40\% (4/10 patients) improvement rate.

Although none of the cases showed complete remission of their tinnitus, this is unsurprising because tVNS paired with notched music therapy is intended to gradually correct the 
maladapted neuronal plasticity in the central auditory system. It is possible that the symptoms would have been cured if the patients received more treatment sessions or if they were followed up for longer. Multiple regression analyses showed that the duration of symptoms was the most important and only factor associated with the overall change in subjective symptom severity (i.e., GI index). In other words, we could predict that the efficacy of the treatment protocol is better in patients with shorter duration of symptoms.

Two patients $(6.6 \%)$ reported mild worsening of symptoms in terms of the GI index. In 4/25 patients (16.0\%), including both patients who reported worsening of symptoms on the GI index, an increase in tinnitus loudness was reported after treatment. In addition, another 4 patients $(16.0 \%)$ reported an increase in tinnitus awareness. This indicates that tVNS paired with notched music therapy could have adverse effects on tinnitus in some patients, although the severity of symptom worsening was not severe.

Ventureyra originally proposed a new concept for tVNS rather than direct nerve stimulation via an implanted cervical electrode to control seizures [23]. Fallgatter, et al. [24] demonstrated the feasibility of a non-invasive technique targeting the sensory auricular branch of the vagus nerve by recording sensory-evoked potentials originating from the brainstem of healthy patients. A subsequent fMRI study revealed that tVNS robustly increased the blood oxygenation level in various cortical regions relating with emotion, and tVNS started to be applied for the treatment affective disorders [13]. Like neuromodulation for the treatment of affective disorders, tVNS paired with notched music is predicted to correct maladapted neural plasticity in the auditory cortex by decreasing central synchronization or increasing lateral inhibition in deafferentiated cortical regions.

In the current study, none of the patients experienced any specific side effects, including changes in heart rate or blood pressure. Although tVNS is expected to selectively stimulate the afferent fibers of the vagus nerve, it may stimulate efferent fibers, which could result in potentially dangerous events such as cardiac bradycardia or hypotension. Because the efferent fibers of the vagus nerve are more likely to be located on the right side, some researchers have recommended that the electrode should be placed on the left side $[25,26]$. For this reason, we placed the electrode on the left auricular concha in all of the patients, regardless of the affected side. We believe that left auricular stimulation can modulate the bilateral auditory cortex via central decussation of the auditory tract covering either side of the lesion. Electrocardiogram and blood pressure were assessed before starting treatment to exclude patients with potential cardiac problems. Patients with asthma were also excluded from our study. An earlier study examining the safety of tVNS also confirmed it had no side effects on heart rhythm for up to 24 weeks of treatment, although two adverse cardiac events, which were very unlikely to be caused by tVNS, occurred in that study [27].

Notched music plays an important role as a carrier to transfer the electrical stimulation of the vagus nerve to the target area (i.e., auditory cortex). Okamoto, et al. [28] reported that listening to spectrally notched music in which a frequency band with a one octave width centered on the individual tinnitus frequency can reduce subjective tinnitus loudness, and evoked activity in the auditory cortex area corresponding to the patient's tinnitus frequency. Audio therapy using music excluding the spectrum corresponding to the tinnitus frequency seems to be a reasonable approach. Because the cortical areas characterized by less lateral inhibition may generate tinnitus via overstimulation or synchronization of neural activity, the target area should be spared from audio stimuli. Instead, it seems reasonable that stimulating the neighboring frequency area of the auditory cortex could induce lateral inhibition of the tinnitus-affected area, and alleviate the tinnitus. However, we do not know the ideal width of the notch yet. If the notch is too wide, it might decrease the effectiveness of lateral inhibition of the target area in the auditory cortex. By contrast, if the notch is too narrow, it might stimulate the tinnitus-generating area. One study has compared the therapeutic effects of notched music with bandwidths of 0.5 , 1 , and 1.5 octaves, and the best results were obtained with the 0.5 octave bandwidth [29]. Therefore, we used a 0.5 octave notch bandwidth in our study. Because it is essential to determine the precise tinnitus frequency to prepare music with a 0.5 octave notch, we excluded patients with inconsistent pitchmatching results in tests performed on 3 days. Further studies would have been necessary to determine the ideal notch width in such patients.

Polak, et al. [14] suggested that the optimal stimulation intensity of tVNS that did not elicit pain was $8 \mathrm{~mA}$, as determined by vagus somatosensory-evoked potentials measured across the scalp. Another study recommended low-frequency stimuli for treating tinnitus, and suggested that frequencies $>100 \mathrm{~Hz}$ should be avoided [30]. Considering these studies, we used alternating currents with a frequency of $25 \mathrm{~Hz}$ and the stimulus intensity was set at the highest level that the patient could endure. The current intensity varied from 1 to 10 $\mathrm{mA}$ among patients, and also varied between sessions in individual patients. Generally, the amplitude tended to increase over time. However, the maximum intensity of the electrical stimulation and the difference between the minimum and maximum intensities were not correlated with the GI index in 
multiple regression analysis. Because the optimal conditions differ among individual laboratories, further studies are needed to determine the optimal conditions for electrical stimulation.

Lehtimäki, et al. [31] reported a successful pilot study of tVNS using a clip-type electrode attached to the patient's left tragus. The mean tinnitus questionnaire score decreased (i.e., improved) following treatment. The auricular branch of the vagus nerve passes through the tympanomastoid fissure and is distributed along part of the skin on the cranial surface of the auricle and posterior part of the external auditory meatus [12]. Therefore, clip-type stimulators attached to the tragus may not cover enough of the area innervated by Arnold's nerve. To address this deficiency, we attached a soft and flexible adhesive electrical pad to the curved skin of the auricle. We believe that our system can stimulate more cutaneous branches of the vagus nerve than does a clip-type electrode. However, vagus nerve branches in the ear canal could not be stimulated using the patch electrode. We might expect to see greater therapeutic effects by using a customized electrode manufactured to fit the individual patient's ear canal and auricle.

The current study has several limitations. First, it is a single-group, uncontrolled pilot study. Therefore, a follow-up study with a control group is needed to confirm the effectiveness of the therapeutic approach used here. Second, we did not use objective tools, such as neuroimaging or electrophysiological studies (e.g., fMRI, EEG, or magnetoencephalogram) to assess the changes in neural activity. Instead, we used patient-reported questionnaires to evaluate the therapeutic outcomes. Third, this study was relatively short, because the outcomes were measured after 10 treatment session. The longer-term outcomes of tVNS paired with notched music therapy are still unclear.

\section{Conclusion}

In this study, we evaluated the feasibility and safety of tVNS paired with notched music therapy to treat patients with refractory chronic tinnitus. tVNS was applied using a pad-type electrode attached to the auricular concha. This therapeutic approach reduced subjective symptom severity, tinnitus intensity, and tinnitus awareness in some of the patients. tVNS is unlikely to elicit unwanted side effects if patients with cardiac arrhythmia or asthma are excluded before treatment, and if the left auricular concha is selectively stimulated.

\section{REFERENCES}

1) Kilgard MP, Merzenich MM. Cortical map reorganization enabled by nucleus basalis activity. Science 1998;279:1714-8.

2) Malow BA, Edwards J, Marzec M, Sagher O, Ross D, Fromes G. Vagus nerve stimulation reduces daytime sleepiness in epilepsy patients. Neurology 2001;57:879-84.

3) Rizzo P, Beelke M, De Carli F, Canovaro P, Nobili L, Robert A, et al. Chronic vagus nerve stimulation improves alertness and reduces rapid eye movement sleep in patients affected by refractory epilepsy. Sleep 2003;26:607-11.

4) Smith DC, Modglin AA, Roosevelt RW, Neese SL, Jensen RA, Browning RA, et al. Electrical stimulation of the vagus nerve enhances cognitive and motor recovery following moderate fluid percussion injury in the rat. J Neurotrauma 2005;22:1485-502.

5) Noreña AJ, Farley BJ. Tinnitus-related neural activity: theories of generation, propagation, and centralization. Hear Res 2013;295:16171.

6) Bartels H, Staal MJ, Albers FW. Tinnitus and neural plasticity of the brain. Otol Neurotol 2007;28:178-84.

7) Vanneste S, Plazier M, van der Loo E, Ost J, Van de Heyning P, De Ridder D. Burst transcranial magnetic stimulation: which tinnitus characteristics influence the amount of transient tinnitus suppression? Eur J Neurol 2010;17:1141-7.

8) Vanneste S, De Ridder D. Differences between a single session and repeated sessions of $1 \mathrm{~Hz}$ TMS by double-cone coil prefrontal stimulation for the improvement of tinnitus. Brain Stimul 2013;6:155-9.

9) Faber M, Vanneste S, Fregni F, De Ridder D. Top down prefrontal affective modulation of tinnitus with multiple sessions of tDCS of dorsolateral prefrontal cortex. Brain Stimul 2012;5:492-8.

10) Friedland DR, Gaggl W, Runge-Samuelson C, Ulmer JL, Kopell $\mathrm{BH}$. Feasibility of auditory cortical stimulation for the treatment of tinnitus. Otol Neurotol 2007;28:1005-12.

11) Engineer ND, Riley JR, Seale JD, Vrana WA, Shetake JA, Sudanagunta SP, et al. Reversing pathological neural activity using targeted plasticity. Nature 2011;470:101-4.

12) Swanson LW. Neuroanatomical Terminology: a lexicon of classical origins and historical foundations. New York, USA: Oxford University Press;2014. p.59.

13) Kraus T, Hösl K, Kiess O, Schanze A, Kornhuber J, Forster C. BOLD fMRI deactivation of limbic and temporal brain structures and mood enhancing effect by transcutaneous vagus nerve stimulation. J Neural Transm (Vienna) 2007;114:1485-93.

14) Polak T, Markulin F, Ehlis AC, Langer JB, Ringel TM, Fallgatter AJ. Far field potentials from brain stem after transcutaneous vagus nerve stimulation: optimization of stimulation and recording parameters. J Neural Transm (Vienna) 2009;116:1237-42.

15) Guy W. Clinical global impressions. In: Guy W, editor. ECDEU Assessment Manual for Psychopharmacology, revised, (DHEW Publ No ADM 76-338). Rockville, MD: National Institute of Mental Health; 1976. p.218-22.

16) Newman CW, Jacobson GP, Spitzer JB. Development of the Tinnitus Handicap Inventory. Arch Otolaryngol Head Neck Surg 1996; 122:143-8.

17) Dobie RA, Sakai CS, Sullivan MD, Katon WJ, Russo J. Antidepressant treatment of tinnitus patients: report of a randomized clinical trial and clinical prediction of benefit. Am J Otol 1993;14:18-23.

18) Duckert LG, Rees TS. Placebo effect in tinnitus management. Otolaryngol Head Neck Surg 1984;92:697-9.

19) Araújo MF, Oliveira CA, Bahmad FM Jr. Intratympanic dexamethasone injections as a treatment for severe, disabling tinnitus: does it work? Arch Otolaryngol Head Neck Surg 2005;131:113-7.

20) De Ridder D, Verstraeten E, Van der Kelen K, De Mulder G, Sunaert $\mathrm{S}$, Verlooy J, et al. Transcranial magnetic stimulation for tinnitus: influence of tinnitus duration on stimulation parameter choice and maximal tinnitus suppression. Otol Neurotol 2005;26:616-9.

21) Kleinjung T, Steffens T, Sand P, Murthum T, Hajak G, Strutz J, et al. Which tinnitus patients benefit from transcranial magnetic stimulation? Otolaryngol Head Neck Surg 2007;137:589-95. 
22) De Ridder D, Vanneste S, Engineer ND, Kilgard MP. Safety and efficacy of vagus nerve stimulation paired with tones for the treatment of tinnitus: a case series. Neuromodulation 2014;17:170-9.

23) Ventureyra EC. Transcutaneous vagus nerve stimulation for partial onset seizure therapy. A new concept. Childs Nerv Syst 2000;16: 101-2.

24) Fallgatter AJ, Neuhauser B, Herrmann MJ, Ehlis AC, Wagener A, Scheuerpflug $\mathrm{P}$, et al. Far field potentials from the brain stem after transcutaneous vagus nerve stimulation. J Neural Transm (Vienna) 2003;110:1437-43.

25) Cristancho P, Cristancho MA, Baltuch GH, Thase ME, O'Reardon JP. Effectiveness and safety of vagus nerve stimulation for severe treatment-resistant major depression in clinical practice after FDA approval: outcomes at 1 year. J Clin Psychiatry 2011;72:1376-82.

26) Nemeroff CB, Mayberg HS, Krahl SE, McNamara J, Frazer A, Henry TR, et al. VNS therapy in treatment-resistant depression: clinical evidence and putative neurobiological mechanisms. Neuropsychopharmacology 2006;31:1345-55.
27) Kreuzer PM, Landgrebe M, Husser O, Resch M, Schecklmann M, Geisreiter F, et al. Transcutaneous vagus nerve stimulation: retrospective assessment of cardiac safety in a pilot study. Front Psychiatry 2012;3:70.

28) Okamoto H, Stracke H, Stoll W, Pantev C. Listening to tailor-made notched music reduces tinnitus loudness and tinnitus-related auditory cortex activity. Proc Natl Acad Sci U S A 2010;107:1207-10.

29) Wunderlich R, Lau P, Stein A, Engell A, Wollbrink A, Rudack C, et al. Impact of Spectral Notch Width on Neurophysiological Plasticity and Clinical Effectiveness of the Tailor-Made Notched Music Training. PLoS One 2015;10:e138595.

30) Hazell JW, Jastreboff PJ, Meerton LE, Conway MJ. Electrical tinnitus suppression: frequency dependence of effects. Audiology 1993; 32:68-77.

31) Lehtimäki J, Hyvärinen P, Ylikoski M, Bergholm M, Mäkelä JP, Aarnisalo A, et al. Transcutaneous vagus nerve stimulation in tinnitus: a pilot study. Acta Otolaryngol 2013;133:378-82. 